\title{
Ueber Subchlorüre und Oxychlorüre des Siliciums;
}

\section{von $L$. Troost und $P$. Hautefeuille ${ }^{*}$ ).}

\section{Darstellung der Siliciumsubchlorüre.}

Wenn man Chlorsiliciumdampf in einer bis nahe zum beginnenden Erweichen erhitzten Porcellanröhre über geschmolzenes Silicium leitet, so erhält man einen Absatz von Silicium und eine Flüssigkeit, die neben unzersetztem Chlorid eine kleine Menge von Subchlorüren enthält. Da bei einmaligem Ueberleiten des Chlorsiliciums nur sehr wenig Subchlorür gewonnen wird, richteten die Verfasser ihren Apparat so ein, dafs dasselbe Chlorsilicium, olne mit der Luft in Berührung zu kommen, mehrmals durch die Röhre getrieben werden konnte. Letztere war zu dem Ende auf beiden Seiten mit gläsernen Recipienten verbunden, die abwechselnd erhitzt und abgekühlt wurden. Jeder der Recipienten war aufserdem mit einer langen, in Quecksilber eintauchenden Röhre versehen, um den Druck anzugeben und die äufsere Luft abzuhalten. Wegen der Erweichung des Porcellans ist nämlich darauf zu achten, dafs der Druck im Inneren dem äufseren Druck immer nahezu gleich bleibe und der Zutritt von Luft würde die Bildung von Oxychloriden veranlassen, die von den Subchlorüren nur äufserst schwierig zu trennen sind. Aber auch mit diesem Apparat war das Ergebnifs nicht befriedigend, denn nach fünf- bis sechsmaligem Durchtreiben des Chlorsiliciums war die Röhre, und zwar gewöhnlich auf beiden Seiten, durch abgeschiedenes Silicium verstopft.

Die Hauptschwierigkeit, welche sich der Gewinnung gröfserer Mengen von Subchlorür entgegenstellt, liegt eben

*) Compt. rend. a 8, 563; vgl. diese Annalen 162, 292. 
darin, dafs die Subchlorüre bei dunkler Rothgluth eine beträchtliche Dissociationstension haben; man mufs daher bei dem beschriebenen Versuch einen sehr raschen Strom von Chlorsiliciumdampf anwenden oder aber fül sehr rasche Abkühlung Sorge tragen. Diese Bedingung läfst sich am Besten herstellen mittelst der Deville'schen heifskalten Röhre *), deren Anwendung eine bessere Ausbeute liefert. Durch die innere, die kalte Röhre, liefs man Wasser von etwa $60^{\circ}$ laufen, um eine Verdichtung von Chlorid auf deren Oberfläche möglichst zu vermeiden. Der Zwischenraum zwischen der heifsen und der kalten Röhre communicirte auf einer Seite mit einem abgekühlten Schlangenrohr, durch welches die aus dem Apparat kommenden Dämpfe fortwährend verdichtet und in das Siedegefäfs zurückgeführt wurden; aus diesem gingen sie auf der anderen Seite wieder in das glühende Rohr über. In dem Apparat fand also eine ununterbrochene Circulation des Chlorsiliciumdampfes statt.

Das Rohproduct einer gut geführten Operation enthält neben viel unzersetztem Chlorid eine gewisse Menge Subchlorür und ein wenig Oxychlorid, dessen Bildung nicht vollständig vermieden werden kann.

Das Bichlorid siedet bei $59^{0}$ und ist daher leicht abzuscheiden. Die Trennung der anderen Verbindungen dagegen erfordert wiederholte fractionirte Destillationen oder Behandlung mit geeigneten Reagentien.

\section{Siliciumsesquichlorür.}

Das Sesquichlorür ist eine farblose leicht bewegliche Flüssigkeit von 1,58 spec. Gewicht bei $0^{0}$. Bei $-14^{0} \mathrm{er}-$ starrt es zu grofsen, der Borsäure ähnlichen Krystallblättern. Es beginnt bei $146^{\circ}$ zu sieden und geht zwischen 146 und

*) Vgl. diese Annalen 1 34, 124. 
$148^{\circ}$ vollständig über. Die Dampfdichte 9,7 , bei $239,4^{0}$ bestimmt, entspricht 4 Volumen für die Formel $\mathrm{Si}_{4} \mathrm{Cl}_{6} *$ ).

Wird das Sesquichlorür an der Luft stark erhitzt, so entzündet sich sein Dampf von selbst; in geschlossenen Gefäfsen beginnt es bei $350^{\circ}$ (siedendes Quecksilber) sich zu zersetzen, jedoch sehr langsam, der Siliciumabsatz innerhalb 24 Stunden ist kaum bestimmbar. Mit der Temperatur wächst aber die Dissociationstension sehr rasch, so dafs sich bei $440^{\circ}$ (Schwefeldampf) innerhalb 24 Stunden von 100 Theilen etwa 90 zersetzen, welches Verhältnifs bei längerem Anhalten der gleichen Temperatur constant bleibt. Bei $800^{\circ}$ ist die Zersetzung nahezu vollständig. Das Sesquichlorür zerfällt dabei gerade auf in Silicium und Bichlorid:

$$
2 \mathrm{Si}_{4} \mathrm{Cl}_{6}=\mathrm{Si}_{2}+3\left(\mathrm{Si}_{2} \mathrm{Cl}_{4}\right) \text {. }
$$

Protochlorür war in den bis zu $440^{\circ}$ erhaltenen Zersetzungsproducten nicht nachzuweisen.

Das Sesquichlorid ist also nur unterhalb $350^{\circ}$ und oberhalb etwa $1000^{\circ}$ beständig. Aus seiner merkwürdigen Eigenschaft, bei einer der Rothgluth nahen Temperatur in Silicium und Siliciumbichlorid zu zerfallen und aus diesen Spaltungsproducten bei einer etwas höheren Temperatur sich wieder zu regeneriren, erklärt sich die Fähigkeit des Chlorids, die Verflüchtigung und Krystallisation des Siliciums zu bewirken.

Nach seiner Zusammensetzung entspricht das Sesquichlorür dem Sesquijodür von Friedel und Ladenburg. Es zersetzt bei Gegenwart von Ammoniak das Wasser in der Kälte und bildet unter Wasserstoffentwickelung Kieselsäure. Mit Wasser allein giebt es bei gewöhnlicher Temperatur das nämliche Sesquioxydhydrat, welches Friedel und Laden burg durch Zersetzung ihres Sesquijodürs mit Wasser erhielten.

Dieses Oxydhydrat reducirt in der Kälte das übermangan-

*) $\mathrm{Si}=14 ; \mathrm{O}=8$ u. s. w. 
saure Kali mit äufserster Raschheit, auch die Chromsäure wird durch dasselbe reducirt, jedoch sehr langsam. Auf Lösungen von Goldchlorid oder seleniger Säure wirkt es bei gewöhnlicher Temperatur nicht ein. Es verbindet sich nicht mit Salzsäure oder Schwefelsäure; Salpetersäure dagegen hält es hartnäckig zurück, selbst beim Auswaschen und Trocknen im Vacuum und Iäfst dieselbe erst entweichen, wenn es bis nahe zu der Temperatur erhitzt wird, bei der es sich unter Erglühen in Kieselsäure verwandelt *).

\section{Siliciumprotochlorïr.}

Diese Flüssigkeit findet man mit dem Sesquichlorid gemengt, namentlich wenn letzteres sich unter solchen Umständen bildete, dafs zugleich Oxychloride von hohem Atomgewicht entstanden. Zusammensetzung, Siedepunkt und Dampfdichte desselben sind schwierig festzustellen, da es äufserst hartnäckig kleine Mengen von Oxychlorid zurückhält.

Sein Dampf entzündet sich an der Luft schon unterhalb der dunklen Rothgluth. Bei Gegenwart von Ammoniak zersetzt es das Wasser und entwickelt dabei im Verhältnifs mehr Wasserstoff als das Sesquichlorür. Mit Wasser von $0^{0}$ giebt es ein Oxydhydrat, welches sich von dem erwähnten Sesquioxydhydrat dadurch unterscheidet, dafs es nicht allein übermangansaures Kali und Chromsäure reducirt, sondern auch aus Goldchlorid metallisches Gold und aus einer wässerigen Lösung von seleniger Säure rothes Selen ausscheidet. Aufserdem scheint es sich gegen starke Säuren als Basis zu verhalten.

\section{Siliciumsubfuorür.}

Diese Verbindung entsteht und verhält sich ganz analog

*) In seinem Verhalten zu Säuren zeigt dieses Oxyd eine gewisse Aehnlichkeit mit den Säuren des Tantals, Niobs, Titans und selbst mit der Zinnsäure. 
dem Sesquichlorür und wird daher wie dieses am Besten unter Anwendung der heifskalten Röhre dargestellt.

Es ist ein weifser sehr fein zertheilter Staub, zersetzt das Wasser bei Gegenwart von Ammoniak unter Wasserstoffentwickelung und giebt mit Wasser von $0^{\circ}$ ein Oxydhydrat, welches sich gegen Oxydationsmittel wie das Sesquioxydhydrat verbält ; seine Zusammensetzung entspricht mithin wahrscheinlich der des Sesquichlorids, was jedoch durch Analysen noch nicht festgestellt werden konnte.

\section{Bildung und Darstellung der Siliciumoxychloride.}

Bei der Sublimation von Silicium mittelst Siliciumchlorid bildet sich immer das schon durch Friedel und Ladenburg bekannte Oxychlorid $\mathrm{Si}_{4} \mathrm{O}_{2} \mathrm{Cl}_{6}$, daneben beobachtet man aber auch weniger flüchtige Oxychloride von höherem Atomgewicht.

Die Vertretung von Sauerstoff durch Chlor, welcher diese Oxychloride ihre Entstehung verdanken, geht in vielen Fällen mit aufserordentlicher Leichtigkeit von Statten; sie erfolgt, wie die zur Darstellung der Oxychloride angestellten Versuche ergeben haben, durch directe Einwirkung des Sauerstoffs auf die Chloride oder das bekannte Oxychlorid. Dafs der Sauerstoff dieser Oxychloride nicht, wie Friedel und Laden burg annehmen, aus dem Material der Gefäfse stammt, ergiebt sich schon daraus, dafs man die Chlorüre fast gänzlich frei von Oxychlorid erhält, wenn man bei der Zersetzung des Bichlorids durch geschmolzenes Silicium jeden Luftzutritt mit der gröfsten Sorgfalt verhütet; es wird aber auch schon dadurch ganz unzweideutig bewiesen, dafs die gleichen Oxychloride bei Einwirkung des Inductionsfunkens auf ein Gemisch von Sauerstoff und Chlorid oder Oxychlorid gebildet werden, denn unter diesen Umständen ist eine Mitbetheiligung der Gefälssubstanz selbstverständlich ausgeschlossen. 
Bei diesem Versuch eben wurden jene neuen Körper zuerst beobachtet. Vortheilhafter erhält man dieselben, wenn man ein Gemenge von Sauerstoff mit dem Dampf des Oxychlorids $\mathrm{Si}_{4} \mathrm{O}_{2} \mathrm{Cl}_{6}$ durch eine mit Porcellanstückchen gefüllte Röhre leitet, welche in einem Gasofen erhitzt wird. Man richtet den Apparat so ein, dafs man das Gasgemisch mehrmals ohne Verlust durch die Röhre abwechselnd hin- und hertreiben kann. Schliefslich erhält man eine Flüssigkeit, welche aufser dem Ueberschufs des angewendeten Oxychlorids eine ganze Reihe von Siliciumoxychloriden enthält. Mit der genaueren Untersuchung dieser Körper noch beschäftigt, geben die Verfasser vorerst nur die Zusammensetzung und wichtigsten physikalischen Eigenschaften derselben an.

Das erste der neuen Oxychloride ist eine leicht bewegliche Fiüssigkeit, die bei 152 bis $\mathbf{1 5 4}^{0}$ siedet. Ilıre Zusammensetzung entspricht der Formel $\mathrm{Si}_{4} \mathrm{O}_{3} \mathrm{Cl}_{5}$. Die bei $440^{\circ}$ bestimmte Dampfdichte kommt der berechneten nahe genug, um sicher zu sein, dafs die gegebene Formel 4 Vol. Dampf entspricht.

Gegen $200^{\circ}$ destillirt mit nahezu constantem Siedepunkt ein zweites Oxychlorid, dem nach der Analyse die Zusammensetzung $\mathrm{Si}_{4} \mathrm{O}_{4} \mathrm{Cl}_{4}$ zukommt; ihre Dampfdichte wurde bei $440^{\circ}$ $=15,5$ gefunden. Diefs führt zu einer Verdoppelung der obigen Formel, denn für $\mathrm{Si}_{8} \mathrm{O}_{8} \mathrm{Cl}_{8}$ berechnet sich, bei einer Condensation auf 4 Vol., die Dampfdichte zu 15,9. Dieses Oxychlorid giebt interessante Producte, namentlich mit Alkohol und Ammoniakgas.

Ein drittes Oxychlorid ist eine gegen $300^{\circ}$ siedende Flüssigkeit von der Zusammensetzung $\mathrm{Si}_{4} \mathrm{O}_{5} \mathrm{Cl}_{3}$. Die Dampfdichte wurde bei $440^{\circ}=31,2$ gefunden, wonach die Formel zu vervierfachen wäre. Für $\mathrm{Si}_{16} \mathrm{O}_{20} \mathrm{Cl}_{12}$ berechnet sich die Dampfdichte zu 28,2; die Differenz weist darauf hin, dafs der Körper noch Spuren des folgenden Oxychlorids enthielt.

Dieses vierte 0xychlorid ist eine ölige Flüssigkeit; es 
wird unter $0^{\circ}$ breiartig, siedet oberhalb $400^{\circ}$ und hat die $\mathrm{Zu}-$ sammensetzung $\mathrm{Si}_{4} \mathrm{O}_{6} \mathrm{Cl}_{2}$; seine Dampfdichte konnte noch nicht bestimmt werden.

Endlich wurde auch ein festes Oxychlorid aufgefunden, welches bei $440^{\circ}$ noch nicht schmilzt. Seine Zusammensetzung entspricht der Formel $\mathrm{Si}_{4} \mathrm{O}_{7} \mathrm{Cl}$. In den vorerwähnten $\mathrm{Oxy}-$ chloriden ist es löslich; diese Lösung wird opalartig, wenn man sie durch Destillation concentrirt.

Die Verfasser stellen die Oxychloride des Siliciums schliefslich in folgender Reihe zusammen :

$\begin{array}{ccr}\text { Einfachste } & \text { Formel 4 Vol. } & \\ \mathrm{Formel} & \text { entsprechend } & \text { Siedepunkt } \\ \left.\mathrm{Si}_{4} \mathrm{OCl}_{7}{ }^{*}\right) & - & \text { - } \\ \mathrm{Si}_{4} \mathrm{O}_{2} \mathrm{Cl}_{6} & \mathrm{Si}_{4} \mathrm{O}_{2} \mathrm{Cl}_{6} & 136 \text { bis } 139^{\circ} \\ \mathrm{Si}_{4} \mathrm{O}_{3} \mathrm{Cl}_{5} & \mathrm{Si}_{4} \mathrm{O}_{3} \mathrm{Cl}_{5} & 152 \text { bis } 154^{\circ} \\ \mathrm{Si}_{4} \mathrm{O}_{4} \mathrm{Cl}_{4} & \mathrm{Si}_{8} \mathrm{O}_{8} \mathrm{Cl}_{8} & 198 \text { bis } 202^{\circ} \\ \mathrm{Si}_{4} \mathrm{O}_{5} \mathrm{Cl}_{3} & \mathrm{Si}_{16} \mathrm{O}_{20} \mathrm{Cl}_{12} & \text { gegen } 300^{\circ} \\ \mathrm{Si}_{4} \mathrm{O}_{6} \mathrm{Cl}_{2} & - & \text { iber } 400^{\circ} \\ \mathrm{Si}_{4} \mathrm{O}_{7} \mathrm{Cl} & - & \text { fest bei } 440^{\circ} .\end{array}$

Oxychloride des Bors, Titans und Zirkonizms.

In derselben Weise, durch directe Einwirkung des Sauerstoffs auf die Chloride, haben die Verfasser mehrere $0 x y-$ chloride der genannten Elemente erhalten. Das Zirkoniumoxychlorid, welches sich unter den geschilderten Versuchsbedingungen am Leichtesten bildet, ist fest, flüchtig und hat die Zusammensetzung $\mathrm{Zr}_{4} \mathrm{O}_{2} \mathrm{Cl}_{6}$; es entspricht demjenigen Siliciumoxychlorid, welches gleichfalls in reichlichster Menge entsteht. Von den Oxychloriden des Titans bildet sich am Leichtesten ein festes, dessen Zusammensctzung der Formel $\mathrm{Ti}_{4} \mathrm{O}_{6} \mathrm{Cl}_{2}$ entspricht. Von den Oxychloriden des Bors endlich haben die Verfasser nur festgestellt, dafs dieselben in gleicher Weise entstehen; ihre Abscheidung mufste, um bei dem niederen Siedepunkt des Chlorbors $\left(17^{\circ}\right)$ nicht allzugrofse Verluste zu erleiden, auf die kältere Jahreszeit verscholen werden.

Aus der näheren Untersuchung der neuen Verbindungen erwarten die Verfasser die Entwickelung intcressanter Beziehungen zwischen den Eigenschaften des Bors und Siliciums und denen des Kohlenstoffs einerseits, andererseits denen von Titan und Zirkonium.

*) Ein um $125^{\circ}$ herum siedendes Oxychlorid zeigte die Zusammensetzung $\mathrm{Si}_{4} \mathrm{OCl}_{7}$; seine Menge war zu gering, um Siedepunkt und Dampfidichte zu bestimmen. 